\title{
ARTICLE
}

\section{Development of Accurate Radioactivity Assessment System for Radiation Survey with Various Detectors}

\author{
Osamu KURIHARA ${ }^{1,2 *}$, Eunjoo KIM ${ }^{2}$, Junichi UEDA ${ }^{2}$, Yuji YAMADA ${ }^{2}$, \\ Hiroko $\mathrm{KIDO}^{3}$, Tomomi OGURI ${ }^{3}$, Shintaro $\mathrm{NEMOTO}^{3}$, Makoto NEMOTO ${ }^{3}$, and Makoto AKASHI ${ }^{2}$ \\ ${ }^{1}$ Japan Atomic Energy Agency, Tokai-mura, Naka-gun, Ibaraki 319-1194, Japan \\ ${ }^{2}$ National Institute of Radiological Sciences, Inage-ku, Chiba-shi, Chiba 263-8555, Japan \\ ${ }^{3}$ Visible Information Center, Tokai-mura, Naka-gun, Ibaraki 319-1192, Japan
}

\begin{abstract}
In response to requests from the sites of radiation emergency medicine, we developed a prototype of a computer system for assessing radioactive contaminants remaining in a wound or on the skin surface. This system implements numerical calibration of detectors measuring the contamination using a Monte Carlo simulation-based radiation transport code (MCNPX), coupled to a numerical phantom modeling the contaminants and the surrounding tissue. With the help of functional graphical user interfaces (GUIs) provided by the system, a user can perform desired simulations without complicated procedures to prepare input files for MCNPX. Benchmark calculations of MCNPX were conducted to verify the simulations and adjust detection-related parameter values not sufficiently provided by suppliers. The developed system should aid in making better assessments of the radiological contamination.
\end{abstract}

\section{KEYWORDS: radioactive contamination, numerical calibration, Monte Carlo simulation, MCNPX}

\section{Introduction}

Contamination with radioactive materials is one of the possible risks for persons engaged in various radiological operations. The contamination associated with injury is sometimes difficult to completely remove. ${ }^{1)}$ In such a case, it is necessary to conduct both identification of the nuclide(s) and determination of their activity in order to assess the subsequent radiological risk for the person., ${ }^{2,3)}$ In the determination, proper counting efficiency calibration of the detector measuring the contamination is very important. However, one of the troublesome matters in the calibration is to prepare an anthropometric physical phantom simulating the contaminant and the surrounding tissue. Although some phantoms have been developed for other in vivo counting techniques ${ }^{4}$ (e.g., whole-body counting or a certain organ counting), there have been no general phantoms available for the contaminants remaining in a wound or on the skin surface after an accident. The configuration of contamination will vary greatly according to the circumstances, so that it is difficult to decide on a single representative case based on which the anthropometric physical phantom would be designed. The most promising solution for this problem is a numerical calibration technique by means of a Monte Carlo (MC) simulation-based radiation transport code, coupled to a numerical phantom. In recent years, this technique has been well validated, and has proved to be a useful tool for realistic calibration that is difficult to be achieved with a physical phantom ${ }^{5-7)}$. However, there still are few practical tools ${ }^{8)}$ for implementing numerical calibrations, especially for the contamination focused on in the present study, despite an urgent need for such calibration at the sites of radiation emergency medicine.

*Corresponding Author, E-mail:kurihara.osamu@jaea.go.jp

(C) Atomic Energy Society of Japan
The purpose of this study is to propose a basic design for a system practically available for reliable assessments of specific contaminations. A prototype of the system was thus newly developed in the present study. In this paper, we describe the outline of this system and some benchmark results of the MC code, MCNPX ${ }^{\mathrm{TM} 9)}$ used in the system.

\section{Outline of the Developed System}

In the development of the system, we attempted to achieve the followings:

(1) A widely applicable system that can accommodate a variety of detectors used in radiological individual monitoring;

(2) Interactively creating a numerical phantom according to obtained information on the actual distribution of the contaminant;

(3) Using the same counting geometry in the simulation as in the measurement;

(4) Operating the system without complicated procedures to prepare an input file for the MC code.

Although the prototype of the system we made does not fully satisfy the above, most of its basic design has been finalized. The outline of this system is explained below.

A general configuration model used in the system is shown in Fig. 1. The contaminant is described with an assembly of rectangular volume elements (voxels) in source region, which is currently composed of a single material (the soft tissue of the human body ${ }^{10)}$ ). The detector is placed parallel to the top surface of the source region. This system consists of the following three programs: (1) a first program for setting a source distribution, (2) a second program for automatically creating a desired input file for MCNPX, and (3) a last program for displaying output results. These 
programs have functional graphical user interfaces (GUIs) to facilitate their execution. All of the programs were written in C\# 3.0 language alone or together with Fortran 90 language, and can be individually executed on a personal computer operated with Windows ${ }^{\mathrm{TM}}$.

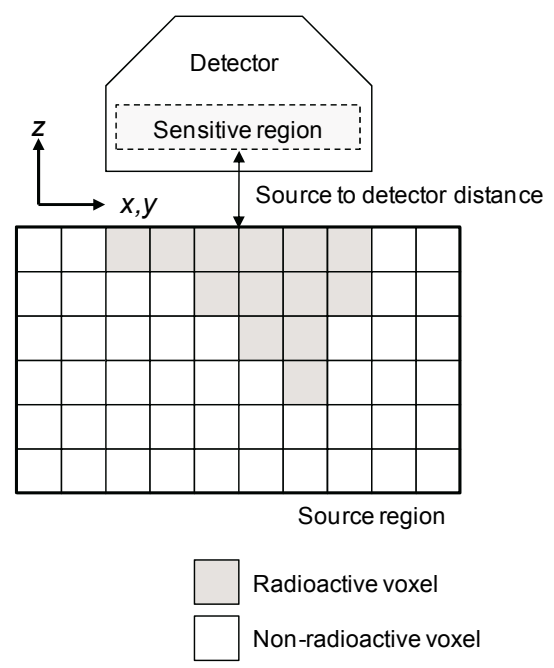

Fig. 1 The general configuration model used in the system.

\section{Program for Setup of Source Distribution}

As described earlier, the contamination to be characterized in this study will vary according to the circumstances and sometimes will have a complicated distribution. It is thus important to take into account how the numerical model of the contamination is created in the system. From the viewpoint of practical use, it is desirable that a user can interactively create as realistic a numerical model of the contamination as possible, and also can implement related procedures easily.

Figure 2 shows the setup display used to make a numerical phantom corresponding to the actual contamination. Each mesh in this figure is one of the voxels belonging to a certain depth layer. A user successively selects meshes and assigns them with relative intensities on the display so that the actual contamination can be sufficiently modeled. For convenience, a drawing tool is available for this procedure. In addition, the user can select one of two segmentations of the source region: a single-layer lattice or a multi-layer lattice. The former is suitable for modeling contaminants adhering to the skin surface; the latter is suitable for modeling contaminants reaching deep into the body.

\section{Program for Calculations with MCNPX}

Fig. 3 shows the display for setting up the desired input file for calculations with MCNPX, together with a display of the next program. On this display, the user selects a desired combination from prepared datasets representing the following items: the radiation, the source distribution, and the detector. The detector position and the number of particles simulated are also set on the same display. After the setup, this program automatically writes the corresponding input file and executes it. The dataset of the source is created by the first program above described. Source descriptions are put in the input file by utilizing the "repeated structure" feature in MCNPX. The other datasets (the radiation and the detector), however, must be directly prepared by the user in the current system. Each data file of these datasets includes energy spectrum (or lines) or configurations of the detector expressed in the MCNPX format.

The system makes use of a pulse height tally estimator (F8) provided by MCNPX to calculate a counting efficiency of a radiation detector. This estimator calculates the energy distribution of pulses generated in the detector by physical interaction with radiations. This estimator can simulate most of the detectors commonly used in radiation monitoring.

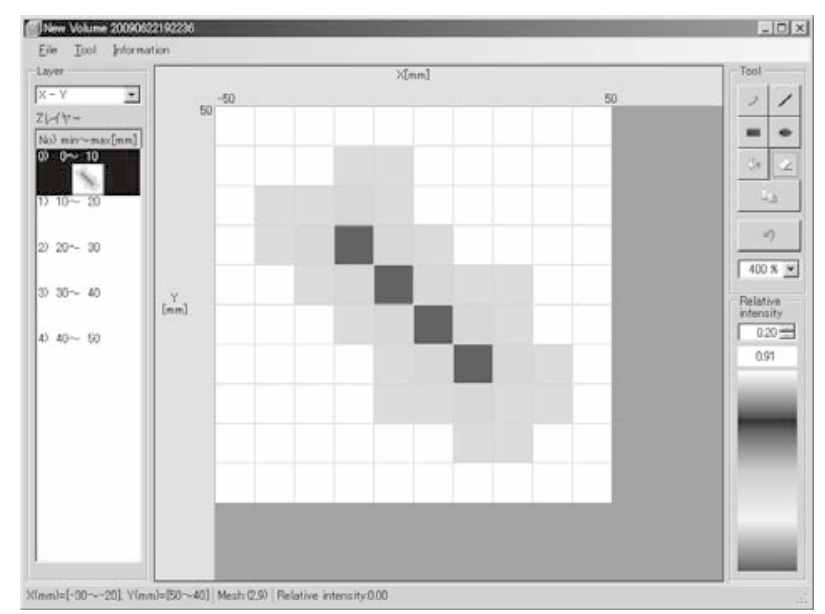

Fig. 2 The setup display for a source distribution.

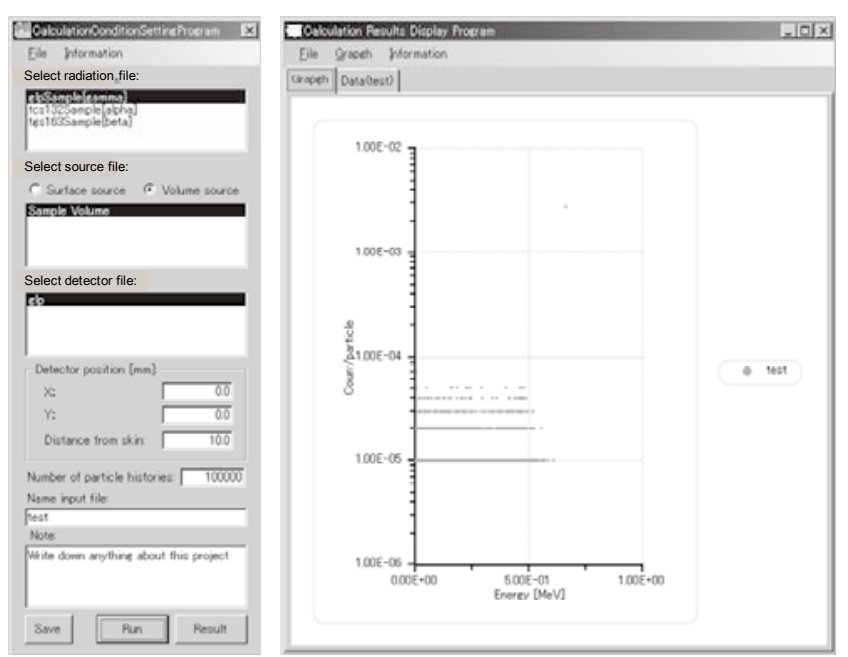

Fig. 3 The setup display for calculations with MCNPX (the left part) and the display of an output result (the right part).

\section{Program for Output Display}

This program displays the results of the pulse height tally estimator in a graph (Fig. 3) or in a table format. The counting efficiency is treated as the sum of the probabilities of the pulses belonging to each of the energy bins above the lowest detectable energy of the detector or each within the energy region of interest. 


\section{Benchmark Calculations of MCNPX}

Benchmark calculations of the MCNPX code were conducted for various detectors that were selected to be incorporated into the prototype system. The aims of the benchmark are twofold: (1) to verify the simulation by comparison with experimental data and (2) to optimize detection-related parameters that are not sufficiently provided by suppliers, such as lowest detectable energy (LDE) or an actual region of detection. Table 1 lists the detectors tested in the present study, together with their brief test results. The major findings of our benchmark calculations are described below.

Table 1 Detectors tested in the benchmark calculations

\begin{tabular}{|c|c|c|}
\hline Detector (for use) & Model (Supplier) & Averaged discrepancy ${ }^{* 1}$ \\
\hline $\begin{array}{l}\mathrm{ZnS}(\mathrm{Ag}) \\
(\alpha \text { survey meter })\end{array}$ & $\begin{array}{l}\text { TCS-232 } \\
\text { (ALOKA, Japan) }\end{array}$ & $10 \%$ for ${ }^{241} \mathrm{Am}^{* 2}$ \\
\hline $\begin{array}{l}\mathrm{ZnS}(\mathrm{Ag}) / \mathrm{plastic} \\
(\alpha / \beta \text { survey meter })\end{array}$ & $\begin{array}{l}\text { TCS-352 } \\
\text { (ALOKA, Japan) }\end{array}$ & $\begin{array}{l}10 \% \text { for }{ }^{241} \mathrm{Am}^{* 2} \\
10 \% \text { for }{ }^{99} \mathrm{Tc},{ }^{36} \mathrm{Cl},{ }^{90} \mathrm{Sr}^{* 3}\end{array}$ \\
\hline $\begin{array}{l}\mathrm{ZnS}(\mathrm{Ag}) \\
\text { ( } \alpha \text { wound monitor) }\end{array}$ & $\begin{array}{l}\text { MCW-102-1 } \\
\text { (ALOKA, Japan) }\end{array}$ & $10 \%$ for ${ }^{241} \mathrm{Am}$ \\
\hline $\begin{array}{l}\text { Plastic } \\
\text { ( } \beta \text { wound monitor) }\end{array}$ & $\begin{array}{l}\text { MCW-102-2 } \\
\text { (ALOKA, Japan) }\end{array}$ & $20 \%$ for ${ }^{99} \mathrm{Tc},{ }^{36} \mathrm{Cl},{ }^{90} \mathrm{Sr}^{* 3}$ \\
\hline $\begin{array}{l}\text { Silicon } \\
(\alpha \text { survey meter) }\end{array}$ & $\begin{array}{l}\text { PASS-100DD } \\
\text { (PROTEC, Japan) }\end{array}$ & $10 \%$ for ${ }^{241} \mathrm{Am}^{* 4}$ \\
\hline $\begin{array}{l}\text { Germanium } \\
(\gamma(X) \text { wound monitor })\end{array}$ & $\begin{array}{l}\text { GLP-36385/10P } \\
\text { (ORTEC, US) }\end{array}$ & $5 \%$ for ${ }^{241} \mathrm{Am}^{* 5}$ \\
\hline $\begin{array}{l}\text { (|Cal-Exp|)/Exp } \times 100 \mathrm{fc} \\
\text { The LDE was set at } 1 . \\
{ }^{3} \text { The result of }{ }^{14} \mathrm{C} \text { was } \\
{ }^{4} \text { The open area ratio of }\end{array}$ & $\begin{array}{l}\text { counting efficienci } \\
\mathrm{MeV} \text { (see text). } \\
\text { cluded. } \\
\text { e entrance window }\end{array}$ & 85) was considered. \\
\hline
\end{tabular}

\section{Survey Meters for Detecting Surface Contamination}

We calculated counting efficiencies of two survey meters: a $\mathrm{ZnS}(\mathrm{Ag}$ ) survey meter (TCS-232, ALOKA, Japan) and a $\mathrm{ZnS}(\mathrm{Ag}) /$ plastic survey meter (TCS-352, ALOKA, Japan). The former is sensitive to alpha particles and the latter is sensitive to both alpha and beta particles. Comparison of experiments with the calculations was conducted using wide area reference sources $\left({ }^{241} \mathrm{Am},{ }^{14} \mathrm{C},{ }^{99} \mathrm{Tc},{ }^{36} \mathrm{Cl}\right.$ and $\left.{ }^{90} \mathrm{Sr}\right)$. Figure 4 shows the calculation model of one of the survey meters, comprising the minimal structure for radiation detection. The protection lattice in front of the Mylar film needed to be precisely incorporated into the model since it greatly affects the detection.

In setting the benchmarks of alpha-particle measurements with the survey meters, we measured counting efficiencies of these detectors as a function of the source to detector distance (SDD). We then compared corresponding calculated efficiencies with the measured efficiencies. As a result, calculated efficiencies were found to be remarkably overestimated, especially for larger SDD. The actual LDE of these detectors were expected to be above $1 \mathrm{MeV}$, judging from the energy spectrum calculated at the maximum detectable SDD. The value of this LDE was adjusted to 1.5
$\mathrm{MeV}$ in accordance with some additional experiments.

In the benchmarking of beta-particle measurements, we found good agreement between the calculated and observed efficiencies, except for the ${ }^{14} \mathrm{C}$ source. Beta energy spectra used in the simulation were taken from DECDC ${ }^{11)}$. More detailed analysis is however needed, since the MCNP code has a tendency toward slight underestimation.

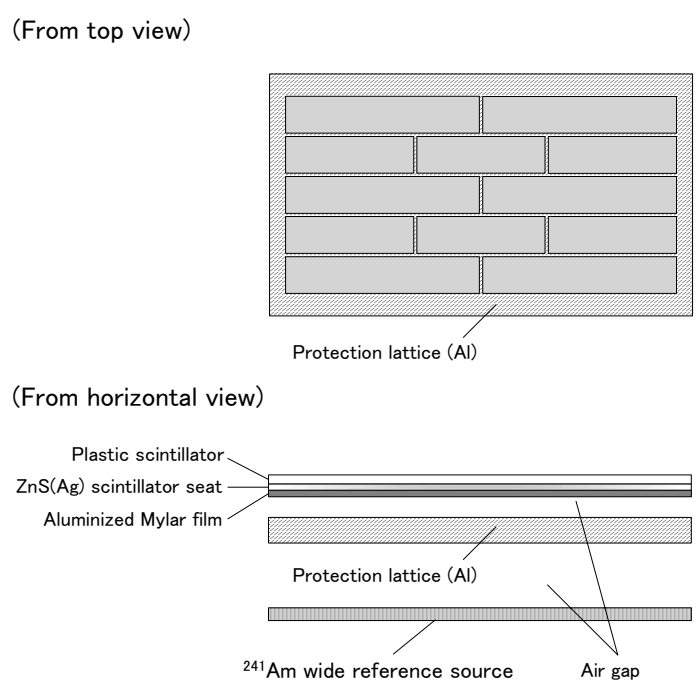

Fig. 4 The calculation model for the survey meter (TCS-352).In the experiment, the air gap distance between the source andthe protection lattice was changed.

\section{Germanium Semiconductor Detector}

We selected a planer-type germanium semiconductor detector (GLP-36385/10P, ORTEC, US) for benchmarking. This germanium detector is designed especially for detecting low energy photons with higher sensitivity. Such a germanium detector is one of the typical detectors used in monitoring of wounds contaminated with plutonium or americium ${ }^{12)}$.

In the simulation, the sensitive region in the germanium crystal should be given the shape which is generally optimum, to obtain better agreement with experimental peak efficiencies. The straightforward method to determine this is sensitivity analysis of the shape of the sensitivity region, assuming insensitive layers attached to the electrodes ${ }^{13,14)}$. The calculation model for this detector is shown in Fig. $\mathbf{5}$ in which a single insensitive layer consisting of germanium is placed on the side toward the entrance window. The experiment was conducted by placing an ${ }^{241} \mathrm{Am}$ point reference source in front of the entrance window. The sensitivity analysis above described was performed keeping the total volume of the crystal constant. Consequently, the optimized thickness of insensitive layer was determined to be $10 \mu \mathrm{m}$ (Table 2). The averaged discrepancy between the calculated and the observed peak efficiencies was within $10 \%$ for the major photons emitted from ${ }^{241} \mathrm{Am}(13.9,17.5,22.0,26.4$ and $59.5 \mathrm{keV})$; in particular, the discrepancy in the peak efficiency was reduced from $80 \%$ to within a few percentage points for $13.9 \mathrm{keV}$ by this optimization. Note that the front insensitive layer which 
was modeled is not effective for adjusting peak efficiencies for higher energy photons (more than $100 \mathrm{keV}$ ). Significant difference was not found between the results with a thick-target bremsstrahlung (TTB) model and with a detailed electron transport model; thus, the former model would be preferable for experiments similar to the above since the latter model is more time-consuming.

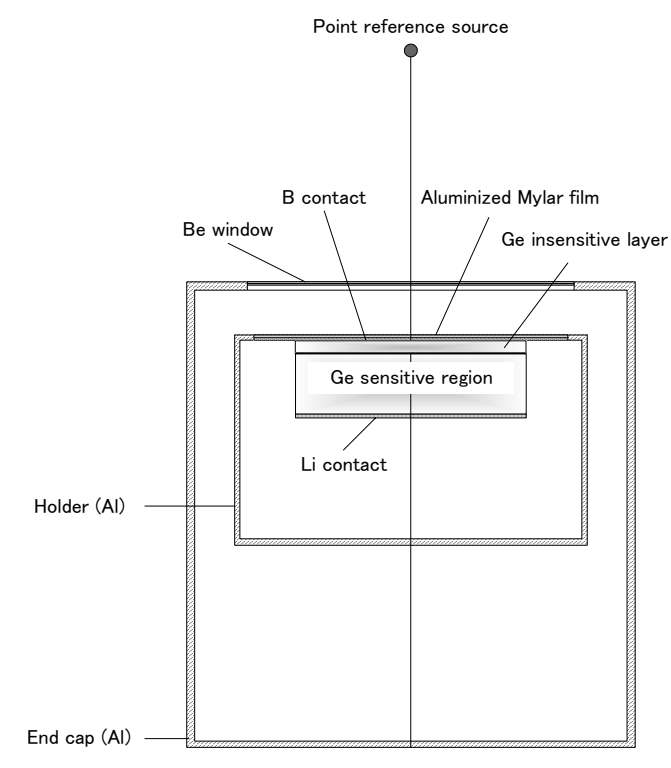

Fig. 5 The calculation model for the germanium semiconductor detector (GLP-36385/10P).

Table 2 Discrepancy between observed and calculated peak efficiencies of ${ }^{241} \mathrm{Am}$ point reference source

\begin{tabular}{ccccc}
\hline \multirow{2}{*}{$\begin{array}{c}\text { Photon } \\
\text { Energy } \\
(\mathrm{keV})\end{array}$} & \multicolumn{4}{c}{$\begin{array}{r}\text { Ratios of calculated to observed peak efficiencies for } \\
\text { different insensitive layer thicknesses }(\mu \mathrm{m})\end{array}$} \\
\cline { 2 - 5 } & 0 & 5 & 10 & 15 \\
\hline \hline 13.9 & 1.75 & 1.30 & 0.97 & 0.72 \\
\hline 17.5 & 1.40 & 1.20 & 1.02 & 0.87 \\
\hline 22.0 & 1.38 & 1.27 & 1.16 & 1.07 \\
\hline 26.4 & 1.01 & 0.96 & 0.91 & 0.86 \\
\hline 59.5 & 0.94 & 0.94 & 0.93 & 0.93 \\
\hline
\end{tabular}

\section{Conclusion and Future Works}

A prototype of a computer system for assessing contaminants remaining in the body was newly developed. This system enables calibrations of detectors measuring the contaminants by means of a Monte Carlo simulation code (MCNPX), coupled to a numerical phantom. User-friendly interfaces provided by the system facilitate procedures for the simulation, such as creating the numerical phantom and writing the input file taking into account actual measurement conditions. Benchmarking of the MCNPX code was done for a variety of detectors in order to confirm the accuracy of their simulations in the system. The basic design of the system was almost finalized, and thus the goal of the next studies is to test the system for more realistic simulated contaminants. Further improvement is being planned for this system.

\section{References}

1) J. J. Jech and J. R. Berry, 1976 Hanford americium exposure incident: external decontamination procedures, Health Physics 45 (4) (1983) 873-881.

2) National Council on Radiation Protection and Measurements, Management of persons accidentally contaminated with radionuclides, NCRP Report 65 seventh reprinting (1997).

3) National Council on Radiation Protection and Measurements, Development of a biokinetic model for radionuclide contaminated wounds and procedures for their assessment, their treatment, dosimetry and treatment, NCRP Report 156 (2006).

4) International Commission on Radiation Units and Measurements, Phantoms and computational models in therapy, diagnosis and protection, ICRU Report 48 (1992).

5) S. Kinase, S. Takagi, H. Noguchi, K. Saito, Application of voxel phantoms and Monte Carlo methods to whole-body counter calibration, Radiat. Prot. Dosim. 125 (1-4) (2007) 189-193.

6) J. G. Hunt, L. Bertelli, B. M. Dantas, E. Lucena, Calibration of in vivo measurement systems and evaluation of lung measurement uncertainties using a voxel phantom, Radiat. Prot. Dosim. 76 (3) (1998) 179-184.

7) M. Moraleda, M. Lis, J. M. Gómez-Ros, M. A. López, A specific voxel phantom for in vivo measurements of ${ }^{241} \mathrm{Am}$ in the knee, Radiat. Prot. Dosim. 127 (1-4) (2007) 258-261.

8) L. de Carlan, I. Aubineau-Lanièce, A. Lemosquet, N. Borissov, J. R. Jourdain, Application of new imaging and calculation techniques to activity and dose assessment in the case of a ${ }^{106} \mathrm{Ru}$ contaminated wound, Radiat. Prot. Dosim. 105 (1-4) (2003) 219-223.

9) D. B. Pelowitz, MCNPX ${ }^{\mathrm{TM}}$ user's manual version 2.5.0, LA-CP-05-0369 (2005).

10) International Commission on Radiation Units and Measurements, Tissue substitutes in radiation dosimetry and measurement, ICRU Report 44 (1989)

11) A. Endo, Y. Yamaguchi, K. F. Eckerman, Nuclear decay data for dose calculation - Revised data of ICRP Publication 38, JAERI 1347 (2005).

12) J. Genicot, F. Hardeman, S. Obserstedt, The assessment of plutonium and americium in contaminated wound with high energy resolution semiconductor detectors, Appl. Radiat. Isot. 46 (3) (1995) 199-203.

13) T. Nakamura, T. Suzuki, Monte Carlo calculation of peak efficiencies of $\mathrm{Ge}(\mathrm{Li})$ and pure $\mathrm{Ge}$ detectors to voluminal sources and comparison with environmental radioactivity measurement, Nucl. Instr. Meth. 205 (1983) 211-218.

14) S. Kamboj, B. Kahn, Evaluating of Monte Carlo simulation of photon counting efficiency for germanium detectors, Health Physics 70 (4) (1996) 512-519. 